\title{
A protocol for isolating Xenopus oocyte nuclear envelope for visualization and characterization by scanning electron microscopy (SEM) or transmission electron microscopy (TEM)
}

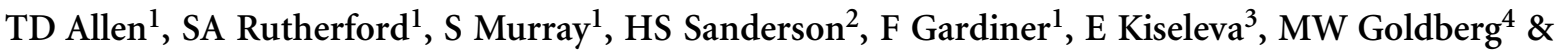 \\ SP Drummond ${ }^{1}$
}

\begin{abstract}
${ }^{1}$ Paterson Institute for Cancer Research, University of Manchester, Wilmslow Road, Withington, Manchester M20 4BX, UK. ${ }^{2}$ Biomedical Research Centre, University of Dundee, Level 5, Ninewells Hospital and Medical School, Dundee DD1 9SY, UK. ${ }^{3}$ Institute of Cytology and Genetics, Russian Academy of Sciences, Novosibirsk 90, 630090, Russia. ${ }^{4}$ School of Biological and Biomedical Sciences, Durham University, South Road, Durham DH1 3LE, UK. Correspondence should be addressed to T.D.A. (tallen@picr.man.ac.uk).
\end{abstract}

Published online 10 May 2007; doi:10.1038/nprot.2007.137

\begin{abstract}
This protocol details methods for the isolation of oocyte nuclear envelopes (NEs) from the African clawed toad Xenopus laevis, immunogold labeling of component proteins and subsequent visualization by field-emission scanning electron microscopy (FESEM) and transmission electron microscopy (TEM). This procedure involves the initial removal of the ovaries from mature female $X$. laevis, the dissection of individual oocytes, then the manual isolation of the giant nucleus and subsequent preparation for high-resolution visualization. Unlike light microscopy, and its derivative technologies, electron microscopy enables 3-5 $\mathrm{nm}$ resolution of nuclear structures, thereby giving unrivalled opportunities for investigation and immunological characterization in situ of nuclear structures and their structural associations. There are a number of stages where samples can be stored, although we recommend that this protocol take no longer than $2 \mathrm{~d}$. Samples processed for FESEM can be stored for weeks under vacuum, allowing considerable time for image acquisition.
\end{abstract}

\section{INTRODUCTION}

Biological material derived from the amphibian Xenopus laevis has been used for decades as an experimental system for the elucidation and characterization of complex cellular events and structures. Study of nuclear structures and dynamics often involves the visualization of protein localization and distribution by conventional direct or indirect immunofluorescence. However, these studies, by their nature, give only limited information on the structural context in which these proteins and complexes are found. Therefore, our laboratory has focused on optimizing protocols for the isolation and high-resolution visualization of oocyte nuclei, nuclei formed in vitro and nuclei derived from both yeast and tissue culture cells ${ }^{1-12}$. Detailed protocols for the visualization of nuclei formed in cell-free extracts of Xenopus eggs and tissue culture cell nuclei ${ }^{13,14}$ can be found in separate manuscripts available at http://www.natureprotocols.com.

The application of field-emission scanning electron microscopy (FESEM) and transmission electron microscopy (TEM) for highresolution visualization of biological specimens has revealed the $3 \mathrm{D}$ structure of macromolecular assemblies such as nuclear pore complexes (NPCs) that mediate the essential, highly dynamic process of nucleo-cytoplasmic transport.

The giant nucleus of Xenopus oocytes (often referred to as the germinal vesicle) is a valuable source of material for visualization and study of nuclear structures at high resolutions, specifically by FESEM and TEM. FESEM yields highly detailed visual information about the surface topology of the sample and is especially useful for determining and examining the structural microenvironment in which specific macromolecular complexes are found. In contrast, TEM involves the sectioning of samples that allows the visualization of internal structures often obscured and thus unavailable for FESEM imaging. However, such sample sectioning generates only a 2D image of the sample, although 3D images of the sample can be generated from serial sectioning and image reconstitution using appropriate computer software (e.g., Reconstruc ${ }^{15}$ ). The nuclei of Xenopus oocytes can be manually dissected and are a rich source of NPCs with approximately 50 million NPCs per nucleus, in contrast to approximately 5,000 in the nuclear envelope (NE) of a somatic cell nucleus. These oocyte nuclei have proved to be important in determining the component structures of the NPC and their associations with the NE and underlying nuclear lamina ${ }^{4-6}$.

Interestingly, comparative 3D cryo-EM mapping studies of the yeast and vertebrate NPC has revealed a striking conservation in structure $^{3}$, thereby supporting the cross-species validity of the ultrastructural data generated by high-resolution visualization of Xenopus oocyte NPCs. The use of immunogold labeling of defined proteins and protein domains with polyclonal or monoclonal antibodies has yielded a wealth of information on the location of individual proteins within the NPC, their topology and the modular NPC structures they are part of (e.g., the localization of nup153 (ref. 9), nup214 and nup358/RanBP2 (ref. 10)). FESEM allows the visualization of only surface morphologies; however, due to the relatively large size of oocyte nuclei (approximately $4-600 \mu \mathrm{m}$ in diameter), it has been possible to visualize the nuclear interior. Kiseleva et al. ${ }^{8}$ revealed that NPCs are connected by an intra-nuclear actin-dependent filament network that also interacts with Cajal bodies and, to a lesser extent, chromatin. The large amphibian nucleus contains relatively little peripheral chromatin compared with somatic nuclei and, therefore, is ideal for visual investigation 
of internal filamentous structures that would otherwise be obscured by chromosomes. In addition, oocyte nuclei are highly active for transcription and nucleocytoplasmic transport and are relatively easy to use in microinjection procedures for intranuclear addition of exogenous compounds or DNA or RNA constructs.
Here, we detail methods for the visualization of amphibian oocyte nuclei from the removal of the oocytes from the source animal, through the manual dissection of the giant nuclei and then the processing of these nuclei for SEM and TEM with immunogold labeling of component proteins.

\section{MATERIALS}

\section{REAGENTS}

- Acetone (VWR International)

- Argon cylinder, high purity greater than $99.999 \%$ (Air Liquide) ! CAUTION Cylinders should be fitted by suitably trained staff.

- Ringers A (see REAGENT SETUP)

- Ringers B (see REAGENT SETUP)

- Amphibian Ringers (see REAGENT SETUP)

- Calcium chloride (Sigma-Aldrich, cat. no. C3306) (see REAGENT SETUP)

- $\mathrm{CO}_{2}$ cylinder, liquid with dip tube (less than 5 p.p.m. water) (Air Liquide)

I CAUTION Cylinders should be fitted by suitably trained staff.

-Ethanol, 30, 50, 70 and $95 \%$ solutions in $\mathrm{dH}_{2} \mathrm{O}$, and $100 \%$ (VWR

International, cat. no. 101077Y)

- Glutaraldehyde, EM grade (Fluka, cat. no. 49631) (see REAGENT SETUP)

! CAUTION Toxic, observe Material Safety Data Sheet (MSDS) advice.

-HEPES (Sigma-Aldrich, cat. no. H7523) (see REAGENT SETUP)

- Osmium tetroxide, $1 \%$ in $\mathrm{dH}_{2} \mathrm{O}$ (Agar Scientific, cat. no. R1017) ! CAUTION

Toxic, observe MSDS advice.

- Potassium chloride (Sigma-Aldrich, cat. no. P9541) (see REAGENT SETUP)

- Potassium hydroxide, $10 \mathrm{M}$ in $\mathrm{dH}_{2} \mathrm{O}$ (VWR International, cat. no.105021)

! CAUTION Corrosive, observe MSDS advice.

- Sodium carbonate (Sigma-Aldrich, cat. no. S7795) (see REAGENT SETUP)

- Tannic acid (TAAB laboratories, cat. no. T046) (see REAGENT SETUP)

- Uranyl acetate, 1 and $2 \%$ in $\mathrm{dH}_{2} \mathrm{O}$ (Agar Scientific, cat. no. R1260A). Can be

stored for several weeks in the fridge if protected from light ! CAUTION

Toxic, observe MSDS advice.

-X. laevis ! CAUTION General animal husbandry and use must be in

accordance with relevant guidelines and regulations.

- BSA (Sigma-Aldrich, cat. no. A7030) (see REAGENT SETUP)

- Fish skin gelatin (Sigma-Aldrich, cat. no. G7765) (see REAGENT SETUP)

- Gly (Sigma-Aldrich, cat. no. G7403) (see REAGENT SETUP)

- Paraformaldehyde (TAAB laboratories, cat. no. P001/1) (see REAGENT

SETUP) ! CAUTION Harmful, observe MSDS advice.

- Primary antibody, as appropriate to research (commercially available or custom made)

- Secondary antibody 10-nm gold conjugate EM grade, as appropriate for primary antibody (GE Healthcare)

- Agar 100 resin kit (Agar Scientific, cat. no. R1031) (see REAGENT SETUP) ! CAUTION Observe MSDS advice.

- Lead nitrate (Fluka, cat. no. 15334) (see REAGENT SETUP) ! CAUTION

Toxic, observe MSDS advice.

- Citric acid trisodium salt (Sigma-Aldrich, cat. no. C3674) (see REAGENT SETUP)

- Sodium hydroxide (VWR International, cat. no. 106495) (see REAGENT

SETUP) ! CAUTION Corrosive, observe MSDS advice.

- Fixative (see REAGENT SETUP)

\section{EQUIPMENT}

-35-mm Petri dishes biological, e.g., BD Falcon (Biotrace International)

-35-mm Petri dishes tissue culture, e.g., BD Falcon (Biotrace International)

- Basket stem assembly (Leica Microsystems, cat. no. 16709248)

- Bunsen or Butane burner (VWR International) ! CAUTION Follow equipment instructions.

- Coating unit with chromium target capable of depositing 2-3 nm layers of chromium with a grain size of less than $0.4 \mathrm{~nm}$. (e.g., Edwards BOC) (see EQUIPMENT SETUP)

- Critical point dryer (e.g., BAL-TEC, Liechtenstein) (see EQUIPMENT

SETUP) ! CAUTION Follow equipment instructions.

- Dissecting microscope, variable zoom with a black base plate and two

flexible light sources for incident illumination (e.g., Leica Microsystems)

- Dissecting needle (VWR International)

- Fine glass needles (see EQUIPMENT SETUP)

- Disposable baskets, small four divisions (Leica Microsystems, cat. no. 16709298)

- Dumont tweezers 3 Dumostar (Agar Scientific)
- Dumont tweezers 5 Dumostar, 2 pairs (Agar Scientific)

- Glass Pasteur pipettes (VWR International) (see EQUIPMENT SETUP)

- High-resolution scanning electron microscope

- Pegwood sticks (Agar Scientific)

- Rubber pipette filler for Pasteur pipettes. (VWR International)

- Silicon chips $5 \times 5 \mathrm{~mm}^{2}$ (Agar Scientific) (see EQUIPMENT SETUP)

-Vacuum desiccator capable of achieving $1 \times 10^{-2} \mathrm{mbar}$

- Writing diamond (Agar Scientific)

- 90-mm plastic Petri dishes (VWR International)

- Backscattered electron detector fitted to SEM

- Filter paper Whatman grade no. 50 (VWR International)

- Nescofilm (VWR International)

- Copper grids 200 mesh hexagonal (Agar Scientific)

- Dumont tweezers 7 Dumostar (Agar Scientific)

- Embedding oven (TAAB laboratories equipment)

- Glass beakers $50 \mathrm{ml}$ (VWR International)

- Grid box (Agar Scientific)

- Plastic disposable pipettes $2 \mathrm{ml}$ (VWR)

- Polyethylene graduated containers with caps (Agar Scientific)

- TEM

- Ultramicrotome (Leica)

REAGENT SETUP

5:1/HEPES buffer $83 \mathrm{mM} \mathrm{KCl}, 17 \mathrm{mM} \mathrm{NaCl}, 10 \mathrm{mM}$ HEPES in $\mathrm{dH}_{2} \mathrm{O}, \mathrm{pH}$ 7.5. Prepare $1 \mathrm{M}$ solutions of $\mathrm{KCl}, \mathrm{NaCl}$ and HEPES and add in appropriate volumes with $\mathrm{dH}_{2} \mathrm{O}$ for final concentrations. Use $10 \mathrm{M} \mathrm{KOH}$ to $\mathrm{pH}$ the HEPES buffer. Ringers A (5× stock) $55 \mathrm{mM} \mathrm{NaCl}, 9.5 \mathrm{mM} \mathrm{KCl}, 5.5 \mathrm{mM} \mathrm{CaCl}_{2}$.

Ringers B $(5 \times$ stock $) \quad 2 \% \mathrm{NaHCO}_{3}$.

Amphibian Ringers $111 \mathrm{mM} \mathrm{NaCl}, 1.9 \mathrm{mM} \mathrm{KCl}, 1.1 \mathrm{mM} \mathrm{CaCl}_{2}, 2.4 \mathrm{mM}$

$\mathrm{NaHCO}_{3}$. Add $200 \mathrm{ml}$ Ringers $\mathrm{A}(5 \times)+750 \mathrm{ml} \mathrm{dH}_{2} \mathrm{O}+10 \mathrm{ml}$ Ringers $\mathrm{B}(5 \times)+$

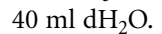

Fixative $2 \%$ Glutaraldehyde, $0.2 \%$ tannic acid in $100 \mathrm{mM}$ HEPES, $\mathrm{pH} 7.4$ freshly prepared and filtered using $0.4 \mu \mathrm{m}$ syringe filter.

BSA $1 \%$ in 5:1/HEPES. Prepare fresh on day of use.

Fish skin gelatin $1 \%$ in 5:1/HEPES. $\triangle$ CRITICAL Prepare fresh on day of use

Fixative $2 \%$ Paraformaldehyde, $0.01 \%$ glutaraldehyde in 5:1/HEPES pH 7.5.

Dissolve paraformaldehyde in buffer by heating up to $80^{\circ} \mathrm{C}$ with agitation, allow to cool; add glutaraldehyde. $\triangle$ CRITICAL Prepare fresh on day of use.

Gly $0.1 \mathrm{M}$ in 5:1/HEPES. $\triangle$ CRITICAL Prepare fresh on day of use.

Agar 100 resin Prepare fresh on day of use from reagents supplied in the kit. Agar 100, dodecinyl succinic anhydride (DDSA), methyl nadic anhydride

(MNA), 2.4.6 tri(dimethylethylaminoethyl) phenol (DMP30) in a ratio of 19.2:10.4:10.4:0.5, respectively. Mix carefully using pegwood sticks avoiding the introduction of air bubbles. Allow to stand for $15 \mathrm{~min}$ before use. Prepare dilutions of one part resin to two parts ethanol and two parts resin to one part ethanol.

Lead citrate Weigh lead nitrate $1.33 \mathrm{~g}$, and citric acid trisodium salt $1.76 \mathrm{~g}$. Place in empty $50-\mathrm{ml}$ flask, add $30 \mathrm{ml}$ water, shake for $1 \mathrm{~min}$, allow to stand for $30 \mathrm{~min}$. Add $8 \mathrm{ml}$ of $1 \mathrm{M}$ sodium hydroxide then add water to make total $50 \mathrm{ml}$. Alternatively, add 1-2 pellets of sodium hydroxide and shake until clear then top up to $50 \mathrm{ml}$. It is not necessary to allow to stand for $30 \mathrm{~min}$ with second method. Store at $4{ }^{\circ} \mathrm{C}$ for as long as the solution remains clear.

EQUIPMENT SETUP

Coating unit Attach argon cylinder and set up to sputter chromium. Further details can be found in ref. 1 .

Critical point dryer Attach liquid $\mathrm{CO}_{2}$ cylinder. Follow manufacturer's instructions for use.

Fine glass needles Prepare from a Pasteur pipette drawn out over a Bunsen flame. The optimal diameter is $0.025 \mathrm{~mm}$ (Fig. 1)

Fine glass pipette Prepare from a Pasteur pipette drawn out over a Bunsen flame. The optimum diameter is $0.5 \mathrm{~mm}$ (Fig. 2; top)

Silicon chips Number chips using the writing diamond. Clean by dipping in a beaker of acetone, and drying with tissue. Store in a covered Petri dish to protect from dust. 


\section{PROCEDURE}

Isolation of nuclear envelope

1) Remove ovary from Xenopus ${ }^{16}$.

$\triangle$ CRITICAL STEP Observe national legislation for handling animals.

PAUSE POINT Store in amphibian Ringers at $4{ }^{\circ} \mathrm{C}$ for up to 1 week or $20^{\circ} \mathrm{C}$ for up to $1-2 \mathrm{~d}$.

2| Wash oocytes in fresh amphibian Ringers, remove small clusters of approximately 20 oocytes and transfer to 5:1/ HEPES buffer. (Fig. 3) All incubations should be done at room temperature (approximately $20^{\circ} \mathrm{C}$ ) using sufficient volumes to prevent the oocytes from drying out.

3| Transfer the oocytes to a 35-mm Petri dish containing fresh 5:1/HEPES buffer and observe under a dissecting microscope with fibre-optic illumination. Carry out all subsequent manipulations under the binoculars. Select oocytes at the required stage of development, stage 5 or 6, 0.8-1.0 mm diameter (see Fig. 4). Use no. 5 tweezers to manipulate the oocytes (Supplementary Video 1 online).

4 Pierce the oocyte with a dissecting needle, avoiding the center of the dark hemisphere where the nucleus is located. The nucleus, visible as a transparent sphere, will be extruded with the cytoplasm (Supplementary Video 1).

? TROUBLESHOOTING

5| Clean the nucleus of excess cytoplasm by pipetting carefully up and down in a fine glass pipette that has an internal diameter slightly larger than the nucleus (Supplementary Video 1).

$\triangle$ CRITICAL STEP Do not allow any air bubbles to touch the nucleus, or else it will lyse and disappear.

? TROUBLESHOOTING

6| For FESEM, carefully transfer the nucleus to a silicon chip (shiny side) in fresh 5:1/HEPES buffer in an adjacent Petri dish (Supplementary Video 1). For TEM, transfer the nucleus to 35-mm tissue culture Petri dishes. ? TROUBLESHOOTING

7| Allow the nucleus to settle. It will attach to the surface of the chip (or Petri dish) within a few seconds. Pipette the buffer against the nucleus very gently to remove any remaining cytoplasm. Carefully break open the nucleus using fine glass needles and continue to pipette the buffer against the NE to remove the nuclear contents. The envelope will attach to the chip (or Petri dish). Use the fine glass needles to further spread and affix the envelope (Supplementary Video 1).

$\triangle$ CRITICAL STEP With practice, it will be possible to attach more than one NE to each chip, but one per chip is advisable for beginners.

$\triangle$ CRITICAL STEP Ensure that there is sufficient buffer above the NE, and cover the dish with its lid to prevent the envelope from drying out.

PAUSE POINT Store NEs at room temperature (approximately $20^{\circ} \mathrm{C}$ ) until a sufficient number has been processed. ? TROUBLESHOOTING

8| Repeat Steps 4-7 until the required number of NEs have been obtained. At least two envelopes per subsequent treatment up to a maximum of 16 chips would be a reasonable number to process at one time. If immunolabeling is required, follow the protocol outlined in Box 1 (Fig. 5) before proceeding to Step 9. Otherwise, continue immediately with Step 9.

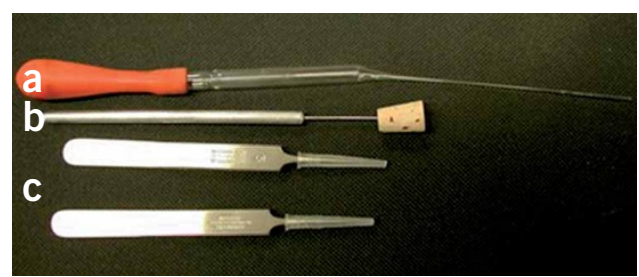

Figure 2 | Tools for manipulating oocytes. (a) Fine glass pipette prepared from a Pasteur pipette drawn out over a Bunsen flame, (b) dissecting needle and (c) Dumont forceps no. 5 (Dumostar).

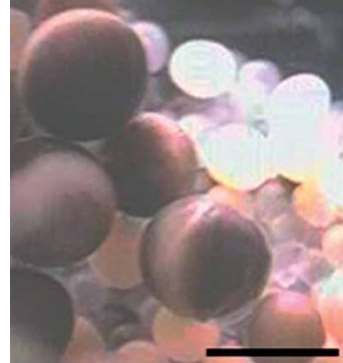

Figure 3 | Xenopus laevis ovary showing different stages of oocyte development. Scale bar $=1 \mathrm{~mm}$. 


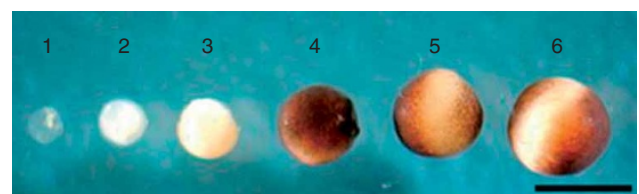

Figure 4 | Xenopus laevis oocytes showing developmental stages 1-6. Stage numbers are marked above the relevant oocyte. Scale bar $=1 \mathrm{~mm}$.

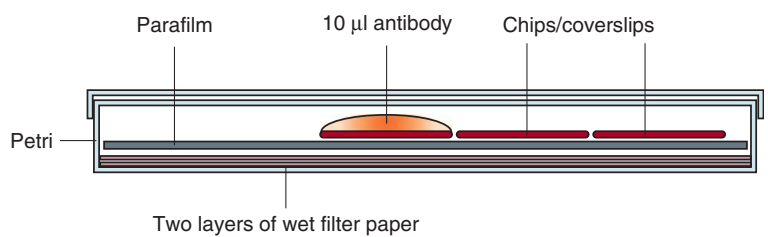

Figure 5 | Wet chamber for immunolabeling steps.

9| Transfer the chip with NE (quickly without air drying) to a $0.5-\mathrm{ml}$ tube containing fixative (2\% glutaraldehyde, $0.2 \%$ tannic acid in $100 \mathrm{mM}$ HEPES, pH 7.4). NEs attached to Petri dishes should be fixed in situ and subsequent steps carried out within the Petri dish. It is helpful to mark the position of the envelope by circling the area with the writing diamond. Fix for a minimum of $10 \mathrm{~min}$ at room temperature (approximately $20^{\circ} \mathrm{C}$ ). This fixative has been optimized for visualization of NPC ultrastructure by FESEM and also works well for TEM.

$\triangle$ CRITICAL STEP For the following steps, use no. 3 tweezers to transfer chips manually into a Petri dish containing the appropriate reagent. For envelopes attached to Petri dishes, washes are added directly. Keep the NE upward. Do not allow to dry out at any point.

PAUSE POINT Store in fixative at $4^{\circ} \mathrm{C}$ for up to 1 week.

10| Wash briefly in $\mathrm{dH}_{2} \mathrm{O}$.

11| Post fix in aqueous $1 \%$ osmium tetroxide for $10 \mathrm{~min}$. Osmium preserves lipid and phospholipid content and also enhances contrast in the specimen.

12| Wash briefly in $\mathrm{dH}_{2} \mathrm{O}$.

13| Stain for 10 min in $1 \%$ aqueous uranyl acetate. This stains nucleic acids and proteins.

14| To process samples for SEM, follow option A. To process for TEM, follow option B.

(A) Processing for FESEM

(i) Dehydrate through the following series of 2 min ethanol washes: 30, 50, 70, $95(2 \times)$ and $100 \%(3 \times)$.

(ii) Transfer to critical point drying apparatus containing ethanol as intermediate reagent. Use a suitable holding device. A Leica basket stem assembly with small baskets is suitable for the Baltec CPD. (It may be necessary to shorten the stem slightly to fit in the chamber.) Critical point dry from high purity (less than 5 p.p.m. water) liquid $\mathrm{CO}_{2}$.

PAUSE POINT Samples can be stored under vacuum for up to 1 week.

(iii) Coat samples with 3-nm chromium.

PAUSE POINT Samples can be stored under vacuum for several months.

(iv) Visualize by high-resolution SEM (Fig. 6a-f).

- PAUSE POINT Store samples under vacuum for optimal preservation. They should last for several months and can be recoated with chromium if required.

? TROUBLESHOOTING

(B) Processing for TEM

(i) Dehydrate samples with one part resin to two parts ethanol for $1 \mathrm{~h}$.

(ii) Replace with two parts resin to one part ethanol for $1 \mathrm{~h}$.
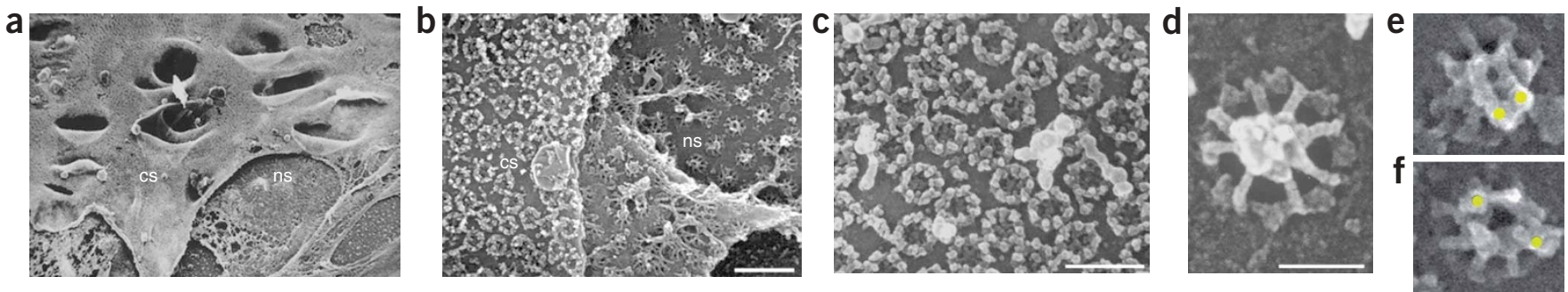

Figure 6 | Field-emission scanning electron microscopy (FESEM) imaging of the oocyte nuclear envelope. (a) Low-power scanning electron microscopy (SEM) of whole oocyte nuclear envelope spread on Si chip. $c s=$ cytoplasmic surface, $\mathrm{ns}=$ nucleoplasmic surface. (b, scale bar $=300 \mathrm{~nm})$ Higher magnification of overlapping area. $(\mathbf{c}$, scale bar $=200 \mathrm{~nm})$ High magnification image of the cytoplasmic face of the NE. (d, scale bar $=50 \mathrm{~nm})$ High magnification of single NE basket. (e, f) Basket labeled with $10 \mathrm{~nm}$ colloidal gold conjugated to nucleoporin-specific antibodies (combined secondary electron and backscattered electron imaging). 
(iii) Replace with $100 \%$ resin for $1 \mathrm{~h}$.

(iv) Replace with fresh $100 \%$ resin for $4 \mathrm{~h}$ or overnight.

(v) Replace with fresh $100 \%$ resin and polymerize in the embedding oven at $60{ }^{\circ} \mathrm{C}$ for $48 \mathrm{~h}$.

(vi) Remove from oven and allow to cool.

- PAUSE POINT Store at room temperature (approximately $20^{\circ} \mathrm{C}$ ) indefinitely.

(vii) Locate the NE in the resin block and trim and cut sections $60-70 \mathrm{~nm}$ thickness using the ultramicrotome. Sections can be cut parallel to or perpendicular to the plane of the embedded resin and hence the envelope.

(viii) Collect sections on 200-mesh hexagonal copper grids. Use Dumont tweezers 7 Dumostar to manipulate the grids.

PAUSE POINT Store dry grids in grid box indefinitely.

(ix) Contrast stain by immersing the grids in drops of lead citrate for $5 \mathrm{~min}$.

(x) Wash by immersion in three beakers of $\mathrm{dH}_{2} \mathrm{O}$, ten dips in each beaker.

(xi) Remove excess $\mathrm{dH}_{2} \mathrm{O}$ with filter paper (number 50).

PAUSE POINT Store dry grid in grid box indefinitely.

(xii) Observe in the TEM (Fig. 7).

? TROUBLESHOOTING
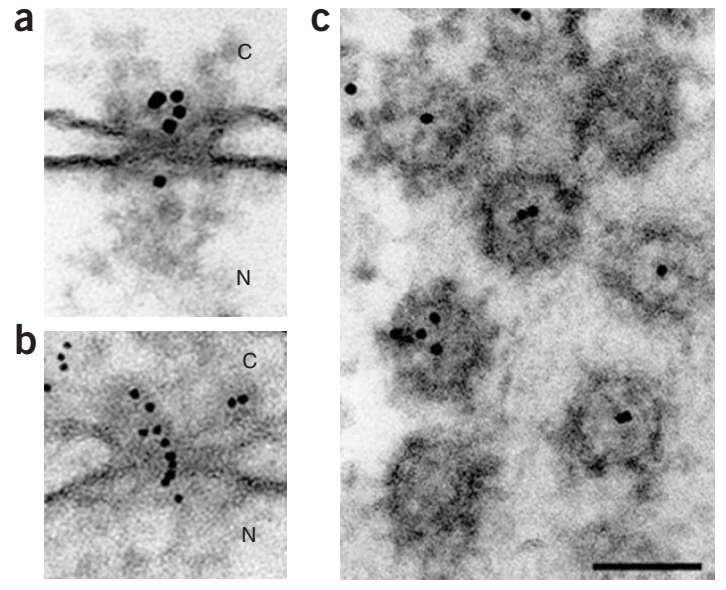

Figure 7 | TEM images of nuclear pore complexes. Complexes are labeled with (a) $10 \mathrm{~nm}$ gold and (b and c) $5 \mathrm{~nm}$ gold. Sections were cut either in perpendicular ( $\mathbf{a}$ and $\mathbf{b}$ ) or in parallel (c) to the nuclear envelope. Uppercase $\mathbf{C}$ denoted the cytoplasmic surface, and $\mathrm{N}$ denoted the nucleoplasmic surface. Scale bar $=100 \mathrm{~nm}$.

\section{TIMING}

Isolation of ovary: Step 1, 1-2 h

Preparation of NEs: Steps 2-7, 2-6 h (depending on number of envelopes required)

Immunolabeling (optional): Step $8,4 \mathrm{~h}$ or overnight (Box 1)

Fixation and washes: Steps 9-13, 40 min

Processing for FESEM: Step 14A(i-ii), 40 min; Step 14A(iii) approximately $1 \mathrm{~h}$ (depending on the coating system used and time required to achieve correct vacuum); Step $14 \mathrm{~A}$ (iv) image acquisition depends on the number of samples

Processing for TEM: Step 14B(i-iv), 8-24 h; Step 14B(v), 48 h; Step 14B(vi-xi) depends on the number of samples; Step $14 \mathrm{~B}(\mathrm{xii})$, image acquisition, depends on number of samples.

\section{? TROUBLESHOOTING}

Troubleshooting advice can be found in Table 1.

\section{BOX 1 | IMMUNOLABELING}

For the following steps, use no. 3 forceps to transfer chips manually into a Petri dish containing the appropriate reagent, or add the solution to envelopes attached directly to Petri dishes. Keep the nuclear envelope upward. Do not allow it to dry out at any point.

1. Fix with $2 \%$ paraformaldehyde, $0.25 \%$ glutaraldehyde (optional) in $5: 1 /$ HEPES buffer for 10 min at room temperature (approximately $20{ }^{\circ} \mathrm{C}$ ) or overnight at $4{ }^{\circ} \mathrm{C}$. Glutaraldehyde gives better ultrastructural preservation, but should be avoided if it inhibits binding of the primary antibody.

2. Wash in $5: 1 /$ HEPES buffer $2 \times 5 \mathrm{~min}$.

3. Transfer to $0.1 \mathrm{M}$ Gly in 5:1/HEPES for 10 min (quenches unreacted aldehyde).

4. Block with $1 \%$ BSA in $5: 1 /$ HEPES buffer for $10-20$ min.

5. Prepare a wet chamber using a 9-cm Petri dish containing wet filter paper with Nescofilm on top.

6. Dilute the primary antibody at an appropriate concentration in 5:1/HEPES.

7. Place chip on dry filter paper to dry the back.

8. Place chip on parafilm in wet chamber and quickly pipette on $10-\mu \mathrm{l}$ antibody, incubate for $1 \mathrm{~h}$ at room temperature.

9. Wash in $5: 1 /$ HEPES buffer $2 \times 10 \mathrm{~min}$.

10. Block with $1 \%$ fish skin gelatin in 5:1/HEPES for $10 \mathrm{~min}$.

11. Dilute the secondary antibody gold conjugate at a concentration of 1 in 10 in 5:1/HEPES.

12. Place chip on dry filter paper to dry the back.

13. Place chip on parafilm in wet chamber and quickly pipette on $10-\mu \mathrm{l}$ antibody, incubate for $1 \mathrm{~h}$ at room temperature.

14. Wash three times for 10 min in 5:1/HEPES.

15. Fix in $2 \%$ glutaraldehyde, $0.2 \%$ tannic acid and $0.1 \mathrm{M} \mathrm{HEPES}$ as described at Step 9 in the main protocol, and continue from there. 
TABLE 1 | Troubleshooting table.

\begin{tabular}{|c|c|c|}
\hline Step & Problem & Possible reason \\
\hline 4 & No nucleus seen & Oocyte may be unhealthy \\
\hline
\end{tabular}

\section{5}

7

Immunolabeling (Box 1)

$14 \mathrm{~A}$ and $\mathrm{B}$

$14 \mathrm{~A}$ and $\mathrm{B}$

$14 \mathrm{~A}$ and $\mathrm{B}$

$14 \mathrm{~A}$ and $\mathrm{B}$
$14 A$ and $B$

No nucleus seen or nucleus lyses on release from oocyte

Nucleus lyses, releasing nuclear contents

Nucleus disappears

Nucleus disappears

Nucleus does not attach to chip (Supplementary Video 1) and/or envelope will not stick

No gold label

No gold label

Nonspecific labeling

Non-specific labeling

Limited gold labeling

$14 \mathrm{~B}$
Gold label is difficult to distinguish from cellular components

Solution

Select only healthy looking oocytes with a clear distinction between their dark and light poles. Avoid the largest stage- 6 oocytes. If all oocytes look mottled and do not release their nuclei, use a different Xenopus. Fresh oocytes are best, although oocytes can be stored in Ringers at $4{ }^{\circ} \mathrm{C}$ for up to 1 week

Apply gentle pressure to the oocyte using fine forceps to extrude the nucleus. Alternatively, tear the oocyte open using forceps positioned at two points on the midline between the dark and light poles. This method can also be used instead of piercing with a needle. Gently tease the cytoplasm apart using the fine glass needles to reveal the nucleus

Nucleus may have been damaged by the needle or forceps, or too much force was applied as it was extruded through the hole made by the needle

Pipetting was too vigorous

Nucleus may have gotten stuck to the inside of the glass pipette

An air bubble has come into contact with the nucleus

Chip may be contaminated with cytoplasm

Oocyte may not have been healthy

NE may not be well attached in Single thickness NEs that are well attached to the chip give

some areas and may have formed best imaging multiple layers

Coating may not be sufficient or Recoating the samples may help has oxidized with time

Fixation may be inappropriate for primary antibody

Insufficient concentration of primary antibody

Insufficient blocking or washing

Nonspecific binding of the gold secondary

Steric hindrance. The gold particle is preventing access to the epitope

Over-staining of the section
Not all nuclear isolation attempts will be successful. Try again with another oocyte. This technique requires a lot of patience and practice. Try a different size of needle and avoid piercing the nucleus, which is located in the dark hemisphere. Do not squeeze the oocyte too much while holding it with the forceps

It may be possible to retrieve the nuclear envelope (NE) and continue with this rather than the intact nucleus

Coat the inside of new pipettes with oocyte cytoplasm before use

Ensure that there is sufficient buffer in the pipette. Do not introduce air bubbles during pipetting. Use a rubber bulb that allows fine control for this technique

Nuclei attach best to clean chips. Try a new chip in fresh buffer

Try a different oocyte. Obtain fresh oocytes if necessary

Check fixation tolerance of antibody. 0mit glutaraldehyde

Increase concentration of primary antibody. EM protocols typically require higher concentrations than for light microscopy

Check that blocking steps are suitable for primary antibody. Adapt protocol if required. Increase washing steps either in number or volume used

Always do a diluent control with no primary antibody to exclude the possibility of nonspecific binding of the gold secondary. Change supplier if this is a problem

Use smaller colloidal gold. 5-nm gold typically gives more labeling than $10 \mathrm{~nm}$, but can be more difficult to detect. 10 $\mathrm{nm}$ can be searched for at $\times 20,000-40,000$ magnification, but $5 \mathrm{~nm}$ usually requires $\times 60,000$

Omit Step 14B(ix). The uranyl acetate in the main protocol may provide sufficient contrast to the sample 
PROTOCOL

\section{ANTICIPATED RESULTS}

It is critical to ensure that the starting material for this protocol is of the highest standard, as this will have the single largest influence on the quality of images that can be captured by either FESEM or TEM. Therefore, it is essential to ensure that animals are kept appropriately. Figure 6 shows several representative images of an isolated and 'spread' oocyte nucleus, indicating the high density of NPCs within the NE and the macromolecular structural components of both the nucleoplasmic and cytoplasmic faces of the NPC. When immunogold labeling specific proteins and protein domains, the secondary electron image (e.g., Fig. 6d-f) is acquired simultaneously and is in exact register with the backscatter image. Consequently, it is straightforward to superimpose the location of the gold particles (via imaging software) as shown in Figure $6 \mathbf{e}$ and $\mathbf{f}$.

When visualizing gold-labeled samples by TEM (Fig. 7), it is important to determine the plane of sectioning to aid orientation and interpretation of the images generated.

\section{Note: Supplementary information is available via the HTML version of this article.}

ACKNOWLEDGMENTS T.D.A., S.A.R., S.M., H.S.S. and S.P.D. would like to acknowledge the support of Cancer Research (CR) UK. E.K. and M.W.G. acknowledge the support of The Wellcome Trust, and F.G. similarly acknowledges the Medical Research Council.

COMPETING INTERESTS STATEMENT The authors declare no competing financial interests.

Published online at http://www.natureprotocols.com

Reprints and permissions information is available online at http://npg.nature.com/ reprintsandpermissions

1. Allen, T.D. et al. Three dimensional surface structure of the nucleus. Methods Cell Biol. 53, 125-138 (1998).

2. Bagley, S., Goldberg, M.W., Cronshaw, J.M., Rutherford, S. \& Allen, T.D. The nuclear pore complex. J. Cell Sci. 113, 3885-3886 (2000).

3. Drummond, S.P., Rutherford, S.A., Sanderson, H.S. \& Allen, T.D. High resolution analysis of mammalian nuclear structure throughout the cell cycle: implications for nuclear pore complex assembly during interphase and mitosis. Can. J. Physiol. Pharmacol. 84, 423-430 (2006).

4. Goldberg, M.W. \& Allen, T.D. High resolution scanning electron microscopy of the nuclear envelope: demonstration of a new, regular, fibrous lattice attached to the baskets of the nucleoplasmic face of the nuclear pores. J. Cell Biol. 119, 1429-1440 (1992).

5. Goldberg, M.W. \& Allen, T.D. The nuclear pore complex: three-dimensional surface structure revealed by field emission, in-lens scanning electron microscopy, with underlying structure uncovered by proteolysis. J. Cell Sci. 106 (Pt 1): 261-274 (1993).
6. Goldberg, M.W. \& Allen, T.D. Structural and functional organization of the nuclear envelope. Curr. Opin. Cell Biol. 7, 301-309 (1995).

7. Kiseleva, E. et al. Yeast nuclear pore complexes have a cytoplasmic ring and internal filaments. J. Struct. Biol. 145, 272-288 (2004).

8. Kiseleva, E. et al. Actin- and protein-4.1-containing filaments link nuclear pore complexes to subnuclear organelles in Xenopus oocyte nuclei. J. Cell Sci. 117 (Pt 12): 2481-2490 (2004).

9. Walther, T.C. et al. The nucleoporin Nup153 is required for nuclear pore basket formation, nuclear pore complex anchoring and import of a subset of nuclear proteins. EMBO J. 20, 5703-5714 (2001).

10. Walther, T.C. et al. The cytoplasmic filaments of the nuclear pore complex are dispensable for selective nuclear protein import. J. Cell Biol. 158, 63-77 (2002).

11. Walther, T.C. et al. The conserved Nup107-160 complex is critical for nuclear pore complex assembly. Cell 113, 195-206 (2003).

12. Zhang, C., Goldberg, M.W., Moore, W.J., Allen, T.D. \& Clarke, P.R. Concentration of Ran on chromatin induces decondensation, nuclear envelope formation and nuclear pore complex assembly. Eur. J. Cell Biol. 81, 623-633 (2002).

13. Allen, T.D. et al. Generation of cell-free extracts of Xenopus eggs and demembranated sperm chromatin for the assembly and isolation of in vitroformed nuclei for western blotting and scanning electron microscopy (SEM). Nat. Protoc. 2, 1173-1177 (2007).

14. Allen, T.D. et al. Visualisation of the nucleus and nuclear envelope in situ by SEM in tissue culture cells. Nat. Protoc. 2, 1180-1184 (2007).

15. Fiala, J.C. Reconstruct: a free editor for serial section microscopy. J. Microsc. 218 (Pt 1): 52-61 (2005).

16. Smith, L.D., Xu, W. \& Varnold, R.L. Oogenesis and oocyte isolation. Methods Cell Biol. 36, 45-60 (1991). 